\title{
Mathematical modeling of COVID-19 spread and stabilization in Madhya Pradesh, India: A fundamental approach
}

Deepak Kumar ( $\boldsymbol{\sigma}$ deepakkumaromre@manit.ac.in )

Maulana Azad National Institute of Technology Bhopal

Vikash Kumar

Maulana Azad National Institute of Technology Bhopal

Vijay Panchore

Maulana Azad National Institute of Technology Bhopal

Ravi Kumar Mandava

Maulana Azad National Institute of Technology Bhopal

Somnath Sarangi

Indian Institute of Technology Patna

\section{Research Article}

Keywords: COVID-19, Modeling, Rough prediction

Posted Date: June 2nd, 2021

DOl: https://doi.org/10.21203/rs.3.rs-574297/v1

License: (9) This work is licensed under a Creative Commons Attribution 4.0 International License. Read Full License 


\section{Mathematical modeling of COVID-19 spread and stabilization in Madhya Pradesh, India: A fundamental approach}

\author{
$1^{\text {st }}$ Deepak Kumar \\ Department of Mechanical Engineering \\ Maulana Azad National Institute of Technology Bhopal \\ Madhya Pradesh, India-462003 \\ deepakkumaromre@manit.ac.in \\ $3^{\text {rd }}$ Vijay Panchore \\ Department of Mechanical Engineering \\ Maulana Azad National Institute of Technology Bhopal \\ Madhya Pradesh, India-462003 \\ vijaypanchore36@manit.ac.in \\ $5^{\text {th }}$ Somnath Sarangi \\ Department of Mechanical Engineering \\ Indian Institute of Technology Patna \\ Bihar, India-801103 \\ somsara@iitp.ac.in
}

\author{
$2^{\text {nd }}$ Vikash Kumar \\ Department of Mechanical Engineering \\ Maulana Azad National Institute of Technology Bhopal \\ Madhya Pradesh, India-462003 \\ vikash@manit.ac.in \\ $4^{\text {th }}$ Ravi Kumar Mandava \\ Department of Mechanical Engineering \\ Maulana Azad National Institute of Technology Bhopal \\ Madhya Pradesh, India-462003 \\ ravikumar1013@manit.ac.in
}

\begin{abstract}
The present study was performed to model a COVID-19 spread and stabilization constraints for the regional people of Madhya Pradesh, India, at the mid-time of the lockdown period. A novel mathematical model was developed to predict the number of days required to break the virus spread chain based on the chain breaking concept of the virus carriers. This model has predicted the very accurate COVID-19 results at a mid-time of the lockdown period. Unfortunately, a good recovery rate was not achieved in Madhya Pradesh as expected by the model to stop the chain of virus spread. Thus, this novel model failed to predict the COVID-19 spread for a large duration. Nevertheless, this novel model has much potential to model the other parallel situations similar to COVID-19 spread.
\end{abstract}

Index Terms-COVID-19, Modeling, Rough prediction.

\section{INTRODUCTION}

A novel coronavirus disease 2019 (COVID-19) is an infectious disease, which is caused by a virus of the corona family named severe acute respiratory syndrome coronavirus 2 (SARS-CoV-2) [1], [2]. It is recently declared a global pandemic by the World Health Organization (WHO) on March 11, 2020. This disease's first case is observed in Wuhan, Hubei province of China, in December 2019 [3]. After that, it rapidly spread out in the whole world. Therefore, the research on COVID-19 in different regions has become a hot topic of research in the current scenario. To fight with this pandemic, besides medical and biological studies on COVID19, modeling studies will also play an equivalent role in

978-1-7281-8586-6/21/\$31.00 C2021 IEEE forecasting the meaningful results to control the virus spread in various levels of the region.

At present, a lot of studies [3]-[10] on this recent epidemic have been reported so many meaningful modeling results based on the different principles of mathematics. Out of them, a few notable studies [3], [4], [7], [8] are primarily aiming the prediction of the virus affected cases. In line with that, Huang et al. [3] reported the mathematical spectrum of common symptoms like fever, cough, myalgia, headache, etc., of 41 patients from Wuhan, China. Next, Hui et al. [4] statistically described several important aspects of MERSCoVepid epidemiology, virology, transmission, diagnosis, etc. Further, Anastassopoulou et al. [7] studied the uncertainty aspects of the available official data. At last, Gamero et al. [8] tried to predict the number of days required to stabilize COVID-19 infection in China.

From the previous studies, we may conclude that there is very limited clinical information of the COVID-19 data sets that are currently available in regard to age, viral kinetics, and transmission route, etc. Therefore, there is a vast scope of new studies aiming for the mathematical description of this COVID-19 pandemic significantly.

The purpose of the present study was to report the mathematical description of the virus chain's outbreak and the prediction of the number of days to stop the chain. Furthermore, the analytical findings were also validated with an official epidemic data set of the people living in Madhya Pradesh, India. The data set was carefully obtained from authoritative 
sources like an English daily newspaper (Deccan Herald) published from The Printers (Mysore) Private Limited and one of the largest circulated newspaper (Navbharat Times) in India published from the Bennett Coleman \& Co. Ltd (BCCL).

Herein, a fundamental solution was analyzed that depends on the awareness of the individuals who follow various healthcare authorities' proper guidelines. The proposed model was applied to two categories: people taking the proper advanced care like Doctors and Nurses, etc. and the routine care like the common men. The affected people were quarantined in the first phase, and others are left instructed for self quarantined. In the second phase for undetected positive persons (hidden carriers), all the detected and undetected people were forcefully quarantined via lock-down to fight with the pandemic. As a result, it was expected that a higher recovery rate should be needed to stop the chain, which is possible only via advanced medical facilities provided by the Government to the people.

\section{Mathematical Modeling}

In this section, a mathematical model is presented to predict the spread and stabilization constraint of COVID-19 infection in a particular society (Madhya Pradesh, India) based on the chain principle of virus carriers.

\section{A. Problem definition}

To define our study system, we may choose the sample of people from a particular society wherein the epidemic of COVID-19 is to be out-broken. Our system can be considered as an open system. This is because the variables are interacting with the environment directly. By adopting the chain breaking concept after a specific time of interval, we may stabilize the situation. For simplification, we ignored the system's inner stricture so that our description of the system can be considered a black box. Our system of the study is considered as a deterministic system. Herein, the determinacy is valid because of the possible variables, and the change of variables is successfully predictable within the acceptable range of certainty. And, the possible uncertainty can be ignored due to the simplification of the problem.

\section{B. Assumptions}

In line with the considered problem, a mathematical model is developed under the following assumptions

- The number of people living in a society are categorized into two different categories of people, namely, people not strongly participating in the virus spreading chain (say infected Doctors and Nurses) and the people strongly participating in the virus spreading chain (say, infected common men).

- The possible efforts to resolve the epidemic problem of COVID-19 are delivered in two different phases. Firstly, the only affected people are quarantined, and others are left instructed for self quarantined. Secondly, all the affected as well as not affected people are quarantined.

\section{A proposed model}

Assume that a COVID-19 infected person comes into a particular society to live therein for an extended period of a time interval, as shown in Figure 1. As we know, a COVID-19 is an infectious disease that may spread easily from an infected person to others from various modes of transfer. Therefore, he may have a strong chance to come into contact with other people living in the same society directly or indirectly.

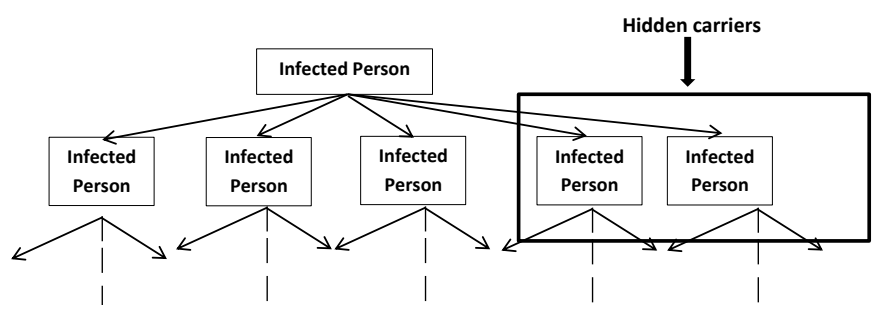

Fig. 1. A tree-diagram for the spread of COVID19 virus infection in a particular society (Madhya Pradesh, India).

Consider that the disease spreads to $x$ number of persons. As per the above assumptions, in the first phase, $\alpha$ persons are quarantined for treatment and will not be in contact with any other person. Next, consider few persons (say $\beta$ ) which are left instructed for self quarantined have not followed the recommendation. Then, $\beta$ is calculated as

$$
\beta=x-\alpha .
$$

These infected persons $\beta$ are the virus carriers of the disease. Each carrier (an infected person) may have a strong chance to infect $x$ other persons in a particular time interval.

In the second phase, it is also possible that few of the persons out of the quarantined persons $\alpha$ sent for treatment are not fully recovered. Then, they may act as an active hidden carrier which is left due to any reason. Now, they will participate in the chain reaction to infect $x$ number of more persons. The hidden carriers (infected people) will increase by a constant number (say $k$ ) because of several reasons like testing, symptoms not shown, hiding travel history, etc., or some newly infected person have come to be involved in the system.

Finally, the number of hidden carriers is increased by $(\beta+$ $k)$. Then, in the next particular time interval, the number of hidden carriers will be increased by $(\beta+2 k)$, and so on till the infection chain is not fully braked. Till that, we do not have any mechanism to identify these hidden carriers. These hidden carriers constantly will remain in contact with all other healthy persons in society. At last, we may obtain a mathematical expression for the total number of infected persons $N_{\text {cases }}$ as

$$
N_{\text {cases }}=1+\left(1+\sum_{i=0}^{i=n}(\beta+i k)\right) x,
$$

wherein $i=0,1,2,3, \ldots . n$. And, $n$ represents a particular time-interval. By applying the summation formula of $n$ terms 
of an arithmetic progression, we may rewrite the above equation (2) as

$$
N_{\text {cases }}=1+x+\frac{n-2}{2}[2 x \beta+(n-3) k x] .
$$

The above relation (3) represents a mathematical model which is able to predict the spread and stabilization constraint of COVID-19 virus infection in a particular society (Madhya Pradesh, India).

\section{RESULTS AND DISCUSSION}

In this section, the mathematical model presented in the previous section II is utilized to predict the number of days required to break the chain of virus spread from hidden carriers (infected people). In addition, the model (3) is validated with the COVID-19 updates in Madhya Pradesh, India, from 1 April 2020 to 15 April 2020.

\section{A. Validation of the proposed model}

To validate the proposed model (2), we use the current updates on COVID-19 in Madhya Pradesh, India, from 1 April 2020 to 15 April 2020. A date-wise data set is sourced from an English daily newspaper (Deccan Herald) published from The Printers (Mysore) Private Limited and one of the largest circulated newspaper (Navbharat Times) in India published from the Bennett Coleman \& Co. Ltd (BCCL). The official data set on COVID-19 in Madhya Pradesh is compared with the model (2) at a particular infection rate $(k x=8.7)$ and $x=1$ as shown in Figure 2. From this Figure 2, we note that the total number of cases $N_{\text {cases }}$ from our model (2) shows a good agreement with the current official updates on COVID19 in Madhya Pradesh, India.

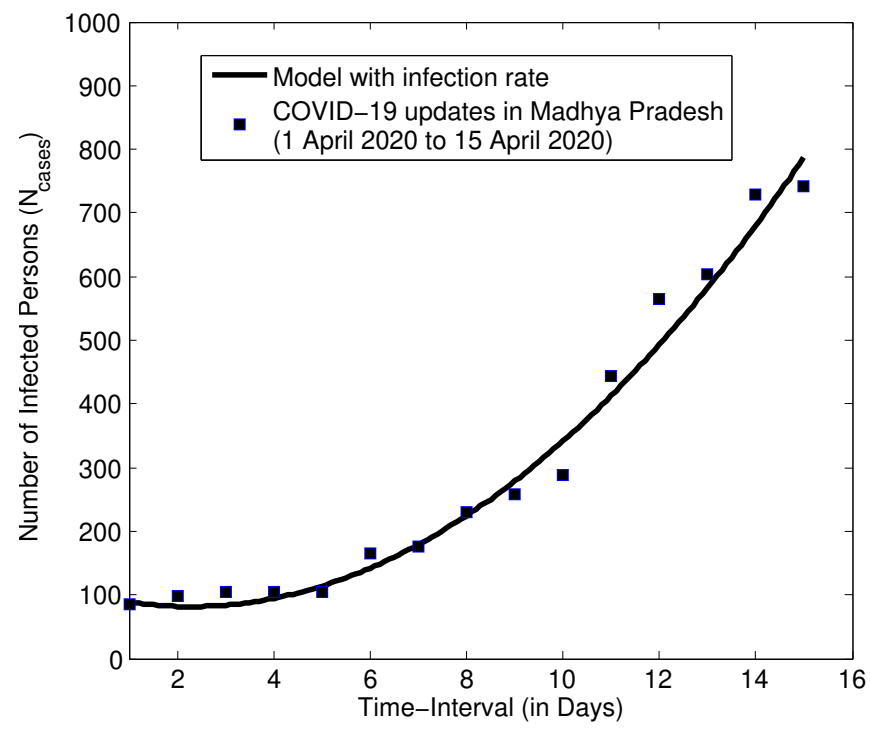

Fig. 2. Validation of proposed model (2) with the COVID-19 updates in Madhya Pradesh, India from 1 April 2020 to 15 April 2020.
B. Time-interval to break the chain of virus spread from hidden carriers

As predicted from the proposed model (2), the total number of COVID-19 infected cases $N_{\text {cases }}$ will increase as time passes. To control this pandemic, the chain of virus infection must be broken to stabilize the number of cases from the officially quarantined people and the unofficially hidden carriers. These hidden carriers are the people who left to self quarantined because they have not shown infection on testing due to various reasons and not followed the guidelines of the healthcare authorities. The only possible solution to break the chain of virus infection is we have that all the detected, as well as undetected people, must be forcefully quarantined via lock-down to fight with the pandemic. In addition, the medical facilities, as well as the awareness on COVID-19, must be enhanced by the Government of Madhya Pradesh via the fast recovery of the infected people to stop the virus from spreading in the state.

Therefore, as per the second phase of our modeling and using the same model (2), we may also predict the number of days required to break the chain of virus spread from hidden carriers (infected people) in the state at different recovery rates (negative and inversely proportional of infection rate). For that, the different curves, as shown in Figure 3, are plotted at different recovery rates. From Figure 3, we note that a higher recovery rate is needed (as expected) to fight with this COVID19.

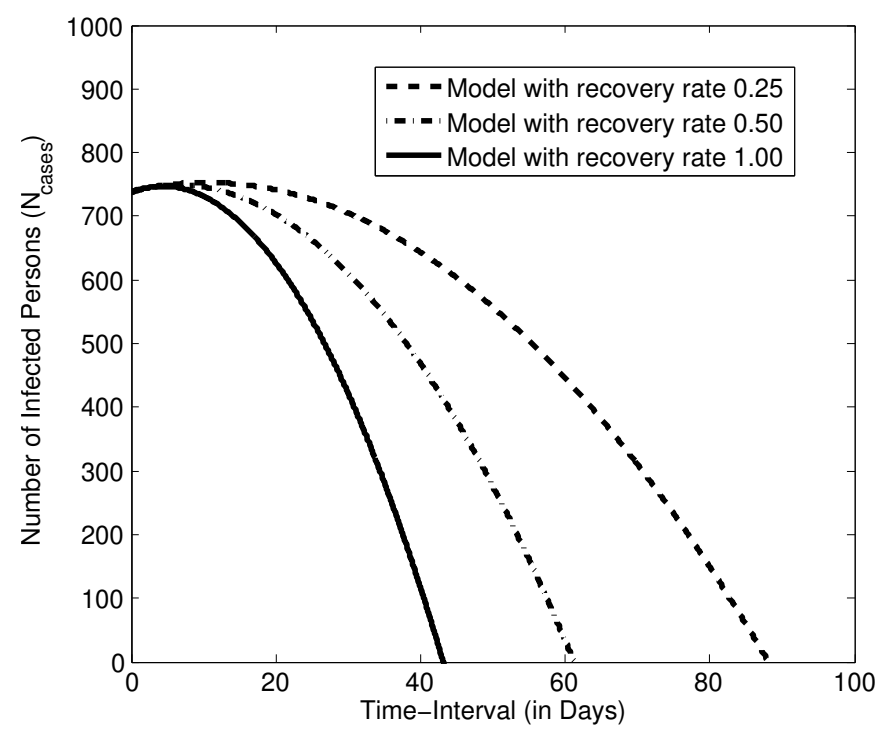

Fig. 3. Number of days required to break the chain of virus spread from hidden carriers (infected people) in Madya Pradesh, India at different recovery rates.

\section{CONCLUding REMARKS}

In the current digital world, various data and information on the novel COVID-19 become available at an unprecedented pace. But, a lot of crucial, as well as simple questions, are unanswered to fight with a pandemic. In line with that, we 
tried to present a mathematical description of COVID-19 to get the answer to the basic questions that came to the mind of people living in a society (Madhya Pradesh, India). For that, we carefully collected the epidemic data from authoritative sources like an English daily newspaper (Deccan Herald) published from The Printers (Mysore) Private Limited and one of the largest circulated newspaper (Navbharat Times) in India published from the Bennett Coleman \& Co. Ltd (BCCL). Then, we used this official data set to model the COVID-19 pandemic and to predict the number of days required to fight this epidemic in the region of Madhya Pradesh, India.

As a result, it was observed that higher recovery rates through medical advancement are very much needed (as expected) to stop this COVID-19 spread. From the viewpoint of the present study, we have believed that the proposed mathematical description of COVID-19 will help to enrich the awareness of the people as well as the Government of Madhya Pradesh.

\section{REFERENCES}

[1] B. Pfefferbaum and C. S. North, "Mental health and the covid-19 pandemic," New England Journal of Medicine, 2020.

[2] G. Chowell and K. Mizumoto, "The covid-19 pandemic in the usa: what might we expect?," The Lancet, vol. 395, no. 10230, pp. 1093-1094, 2020.

[3] C. Huang, Y. Wang, X. Li, L. Ren, J. Zhao, Y. Hu, L. Zhang, G. Fan, J. Xu, X. Gu, et al., "Clinical features of patients infected with 2019 novel coronavirus in wuhan, china," The Lancet, vol. 395, no. 10223, pp. 497-506, 2020.

[4] D. S. Hui, E. I. Azhar, T. A. Madani, F. Ntoumi, R. Kock, O. Dar, G. Ippolito, T. D. Mchugh, Z. A. Memish, C. Drosten, et al., "The continuing 2019-ncov epidemic threat of novel coronaviruses to global health-the latest 2019 novel coronavirus outbreak in wuhan, china," International Journal of Infectious Diseases, vol. 91, p. 264, 2020.

[5] V. M. Corman, O. Landt, M. Kaiser, R. Molenkamp, A. Meijer, D. K. Chu, T. Bleicker, S. Brünink, J. Schneider, M. L. Schmidt, et al., "Detection of 2019 novel coronavirus (2019-ncov) by real-time rt-pcr," Eurosurveillance, vol. 25, no. 3, p. 2000045, 2020.

[6] C. Rothe, M. Schunk, P. Sothmann, G. Bretzel, G. Froeschl, C. Wallrauch, T. Zimmer, V. Thiel, C. Janke, W. Guggemos, et al., "Transmission of 2019-ncov infection from an asymptomatic contact in germany," New England Journal of Medicine, vol. 382, no. 10, pp. 970-971, 2020.

[7] C. Anastassopoulou, L. Russo, A. Tsakris, and C. Siettos, "Data-based analysis," Modelling and Forecasting of the novel Coronavirus (2019nCoV) outbreak. medRxiv, vol. 2020, no. 11.20022186, 2020.

[8] J. Gamero, J. A. Tamayo, and J. A. Martinez-Roman, "Forecast of the evolution of the contagious disease caused by novel coronavirus (2019ncov) in china," arXiv preprint arXiv:2002.04739, 2020.

[9] K. Sarkar, S. Khajanchi, and J. J. Nieto, "Modeling and forecasting the covid-19 pandemic in india," Chaos, Solitons \& Fractals, vol. 139, p. 110049, 2020.

[10] A. Singhal, P. Singh, B. Lall, and S. D. Joshi, "Modeling and prediction of covid-19 pandemic using gaussian mixture model," Chaos, Solitons \& Fractals, vol. 138, p. 110023, 2020. 